\title{
Appropriate Term/Code Not Available Problem Code
}

National Cancer Institute

\section{Source}

National Cancer Institute. Appropriate Term/Code Not Available Problem Code. NCI

Thesaurus. Code C64343.

A device problem is not adequately described by any other term. Note: this code must not be used unless there is no other feasible code. 